Check for updates

Cite this: Analyst, 2021, 146, 6297

\section{Anchoring zinc-doped carbon dots on a paper- based chip for highly sensitive fluorescence detection of copper ions $\uparrow$}

\author{
Qinglan Miao, + $^{\mathrm{a}} \mathrm{Ji}$ Qi, $+^{\mathrm{b}}$ Yuanyuan Li, ${ }^{\mathrm{a}}$ Xinxia Fan, ${ }^{\mathrm{a}}$ Dongmei Deng, ${ }^{\text {a }}$ Xiaoxia Yan, ${ }^{a}$ \\ Haibo $\mathrm{He}^{\mathrm{a}}$ and Liqiang Luo (iD *a
}

\begin{abstract}
In this work, zinc-doped carbon dots (Zn-CDs) were anchored on a three-dimensional wheel type paperbased microfluidic chip, and were decorated with 6-mercaptonicotinic acid (MNA) and L-cysteine (L-Cys) for highly sensitive and rapid fluorescence detection of $\mathrm{Cu}^{2+}$. Zn-CDs were first anchored on paper through the amide bonds between the carboxyl groups of the $Z n-C D s$ and the amino groups of the paper. Afterwards, Zn-CDs were decorated with MNA and L-Cys, effectively preventing the Zn-CDs from aggregation. The nitrogen atom on the pyridine ring and the carboxylic acid groups in MNA and L-Cys Coordinated with $\mathrm{Cu}^{2+}$ to form a nonfluorescent ground-state complex, causing the fluorescence quenching of the $\mathrm{Zn}-\mathrm{CDs}$. The three-dimensional rotary design could simplify the operation process and achieve simultaneous analysis of multiple samples with different concentrations. Under optimal conditions, the fluorescent sensor exhibits linear response for the determination of $\mathrm{Cu}^{2+}$ in the range from 0.1 to $60 \mu \mathrm{g}$ $\mathrm{L}^{-1}$ with the detection limit (LOD) of $0.018 \mu \mathrm{g} \mathrm{L}^{-1}$. The proposed strategy provides a novel way for the highly sensitive detection of $\mathrm{Cu}^{2+}$ in a complex water environment.
\end{abstract}

Received 15th July 2021, Accepted 26th August 2021

DOI: $10.1039 /$ d1an01268a rsc.li/analyst on the carboxyl and amino groups of CDs. Thus, modification is generally achieved through covalent bonds. ${ }^{7}$ In addition, it is difficult to make secondary modifications once the original active groups on the surface of the CDs have been consumed and utilized. If the surface of CDs can contain metal element sites, it will be very easy for CDs to modify groups on the surface through strong coordination bonds between the metal and the groups (such as $-\mathrm{SH},-\mathrm{NH}_{2}$ and $-\mathrm{COOH}$ ), similar to metal nanomaterials. ${ }^{8}$ Metal-doped CDs provide a good opportunity for this. On the other hand, surface modification and heteroatom doping have been developed to enhance the optical and electronic properties of CDs. ${ }^{9,10}$ So far, non-metal heteroatoms doped CDs have been used as the sensing materials for fluorescence detection of metal ions and have broadened the applications in the area of multiplexed detection and bioimaging. ${ }^{11,12}$ However, there are few reports on metal-doped CDs for simplified functional modification and fluorescence analysis. ${ }^{13}$

Fluorescence analysis has been widely applied in biomedicine, biological imaging and diagnostics. ${ }^{14,15}$ The fluorescent paper-based analytical devices ( $\mu$ PADs) based on CDs have found widespread applications due to their portability, low cost, biodegradability and user-friendliness. ${ }^{16}$ CDs are mostly constructed on paper chips by soaking and printing, however, CDs constructed from these simple physical methods are not stable enough. In a previous work, Chen's group reported that 
CdTe quantum dots were grafted onto paper through covalent bonds to achieve paper-based ion imprinted fluorescence sensing for the detection of heavy metal ions. ${ }^{17}$ We hope that CDs can also be stably grafted on the surface of paper through covalent bonds, while retaining their fluorescence properties to the greatest extent and be further modified. We attempted to synthesize paper-based Zn-CDs modified with carboxyl groups which were utilized to form covalent bonds with the amino groups on the surface of the paper. Then, Zn-doped CDs would offer more active sites for functional modification. ${ }^{18,19}$ According to this idea, a stable fluorescence sensing paperbased chip was prepared to achieve fluorescence analysis.

Copper is a vital trace element in humans and animals. ${ }^{20}$ However, unbalanced $\mathrm{Cu}$ (II) levels (both deficiency and excess) in the body may cause variations in brain function, metabolic disorders like obesity and diabetes, and neurodegenerative diseases. $^{21-23}$ According to the quality standard of the United States Environmental Protection Agency, the content of $\mathrm{Cu}^{2+}$ should be lower than $1.3 \mathrm{mg} \mathrm{L}^{-1}$ in drinking water. ${ }^{24}$ Thus, it is significant to develop advanced methods for the microanalysis of $\mathrm{Cu}^{2+}$ ions. To date, a number of analytical methods for copper ion detection in trace amounts have been proposed, including electrochemistry, ${ }^{25-27}$ colorimetry, ${ }^{28-30}$ fluorimetry, ${ }^{31-33}$ spectrophotometry, ${ }^{34}$ etc. Among them, fluorimetry for $\mathrm{Cu}^{2+}$ detection has attracted much attention due to its simplicity, high sensitivity and selectivity. In particular, the fluorescence analysis of copper ions using CD-based $\mu \mathrm{PAD}$ is more sensitive and reliable.

Herein we designed a three-dimensional wheel type $\mu \mathrm{PAD}$ on which zinc-doped carbon dots (Zn-CDs) were grafted by the amide bonds to guarantee the fixing effect. Zn-CDs were decorated with MNA and $\mathrm{L}_{-}$Cys for fluorescence detection of $\mathrm{Cu}^{2+}$. Zn-doping can increase the number of active sites of CDs for the moderate modification of L-Cys and MNA, simplify the reliable secondary modification of CDs, and achieve rapid and sensitive detection of $\mathrm{Cu}^{2+}$. Moreover, the designed threedimensional wheel type paper-based microfluidic chip can easily detect multiple samples. The proposed paper-based fluorescent sensor has also been applied to the detection of $\mathrm{Cu}^{2+}$ in real water samples, indicating great potential in environmental analysis and point-of-care testing (POCT).

\section{Experimental section}

\subsection{Materials}

3-Aminopropyltriethoxysilane (APTES), 3-ethylcarbodiimide hydrochloride (EDC), $\mathrm{N}$-hydroxysuccinimide (NHS), 2- $\mathrm{N}$-morpholinoethanesulfonic acid (MES), and 6-mercaptonicotinic acid (MNA) were obtained from Aladdin (Shanghai, China). Citric acid, zinc acetate, and L-cysteine (L-Cys) were supplied by Sinopharm Reagent (Shanghai, China). Whatman No. 1 chromatography filter paper was procured from GE Company (Shanghai, China). All chemicals were of analytical grade. Milli-Q water (18.25 $\mathrm{M} \Omega \mathrm{cm}$ ) was employed during the whole experimental process.

\subsection{Instruments}

The three-dimensional wheel type $\mu \mathrm{PAD}$ was designed using the FreeHand MX software. Whatman No. 1 chromatography filter paper was printed using a Xerox ColorQube 8560 colour spray wax printer (Fuji, Japan). The fluorescence spectra were obtained with a RF-5301PC fluorescence spectrometer (Shimadzu, Japan). The UV-vis spectra were obtained on a SP-2500 spectrophotometer (Shanghai, China). The morphological structures were recorded by JSM-6700F scanning electron microscopy (SEM, Japan) and JEM-2100F high-resolution transmission electron microscopy (HRTEM, Japan). The elemental compositions were analyzed using an ESCALAB 250Xi X-ray photoelectron spectrometer (XPS, USA). Fourier transform infrared spectra (FT-IR) of samples were recorded using a NEXUS 470 FT-IR spectrometer (Thermo Nicolet, USA).

\subsection{Synthesis of $\mathrm{Zn}-\mathrm{CDS}$}

Zn-CDs were synthesized according to the previously mentioned protocol with slight modification. ${ }^{1}$ Briefly, $10 \mathrm{~g}$ of citric acid and $5 \mathrm{~g}$ of zinc acetate were added into a Teflon-lined stainless-steel autoclave and reacted at $180{ }^{\circ} \mathrm{C}$ for $40 \mathrm{~h}$. Then, Zn-CDs were obtained by oven drying at $60{ }^{\circ} \mathrm{C}$ for $12 \mathrm{~h}$. For comparison, CDs were synthesized by the same procedure in the absence of zinc acetate.

\subsection{Preparation of paper@Zn-CDs}

First, Whatman No. 1 chromatography filter paper $(9 \mathrm{~mm} \times$ $9 \mathrm{~mm}$ ) was pretreated by soaking in $0.2 \mathrm{~mol} \mathrm{~L}^{-1} \mathrm{HCl}$ solution for $30 \mathrm{~min}$, followed by washing three times with ultrapure water to remove excess hydrochloric acid. Then, the pretreated paper was attached to the glass slide and placed into a Petri dish. $40.0 \mathrm{~mL}$ of ethanol/water $(1: 1)$ and $1.5 \mathrm{~mL}$ of APTES were added to the Petri dish to graft amino groups. Afterwards, $8 \mathrm{~mL}$ of $\mathrm{Zn}-\mathrm{CD}$ solution $\left(12.5 \mathrm{mg} \mathrm{mL}^{-1}\right)$ was added to the above Petri dish. Finally, $6 \mathrm{~mL}$ of NHS $\left(10 \mathrm{mg} \mathrm{mL}^{-1}\right)+$ EDC $\left(20 \mathrm{mg} \mathrm{mL}^{-1}\right.$ ) was added and oscillated in the dark for $5 \mathrm{~h}$ to graft $\mathrm{Zn}$-CDs on the surface of the paper.

\subsection{Fabrication of MNA-L-Cys-paper@Zn-CDs}

MNA-L-Cys-paper@Zn-CDs were fabricated based on the reported method. ${ }^{19}$ Briefly, $24 \mathrm{~mL}$ of the mixed solution of MNA ( $2 \mathrm{mg} \mathrm{L}^{-1}$ ) and L-Cys $\left(1.6 \mathrm{mg} \mathrm{L}^{-1}\right)$ was added into a Petri dish. The total volume was made up to $40 \mathrm{~mL}$ by adding water. Then, the prepared paper@Zn-CDs were immersed in the above solution, followed by shaking for $12 \mathrm{~h}$ in the dark to obtain MNA-L-Cys-paper@Zn-CDs. Afterwards, the fabricated MNA-L-Cys-paper@Zn-CDs were washed 3-5 times with water and dried naturally. Finally, the filter paper $(9 \mathrm{~mm} \times 9 \mathrm{~mm})$ was cut into a circle with a diameter of $7 \mathrm{~mm}$, and refrigerated until further use.

\subsection{Design and construction of the three-dimensional wheel type $\mu$ PAD (MNA-L-Cys-paper@Zn-CD $\mu$ PAD)}

The pattern of MNA-L-Cys-paper@Zn-CDs $\mu$ PAD was designed using the Freehand MX software and printed on Whatman No. 
1 chromatography filter paper. The printed paper was placed in a blast drying oven at $100{ }^{\circ} \mathrm{C}$ for $4 \mathrm{~min}$. The wax melted and penetrated into the paper slowly to form a hydrophobic barrier that controlled the sample flow along the hydrophilic "L" channel to the sensing zone by capillary forces.

As seen from Fig. 1A, the $\mu \mathrm{PAD}$ was composed of three layers. The top sampling layer was circular in shape with a diameter of $65 \mathrm{~mm}$, including four sample zones $(3 \mathrm{~mm}$ in diameter), four holes ( $7 \mathrm{~mm}$ in diameter) and eight transporting zones. The middle octagonal layer was composed of eight holes ( $7 \mathrm{~mm}$ in diameter) corresponding to the large circular sample zones of the top sampling layer. The middle isolation layer could effectively prevent samples from flowing to the other sensing zones during rotation. The sensing layer was $97 \mathrm{~mm}$ in diameter at the bottom of the microfluidic chip (Fig. S1†). The sensing zones (diameter $7 \mathrm{~mm}$ ) were covered with 8 prefabricated fluorescence sensors (MNA-L-Cyspaper@Zn-CDs). The determination of copper ions with four different concentrations could be achieved on the sensing zones (Fig. S1C $\dagger$ ).

\subsection{Condition optimization of the MNA-L-Cys-paper@Zn-CD $\mu \mathrm{PAD}$}

To explore the optimal conditions, we investigated the fluorescence intensity changes of the experimental results using different mass ratios of precursors, contents of Zn-CDs, volumes of APTES, volumes of MNA and L-Cys, $\mathrm{pH}$ values, and reaction time, respectively. With other conditions fixed, $10 \mathrm{~g}$ of citric acid was mixed with $0,1,2,3,4$, and $5 \mathrm{~g}$ of zinc acetate in the synthesis of Zn-CDs. For contents of Zn-CDs, we used

(A)

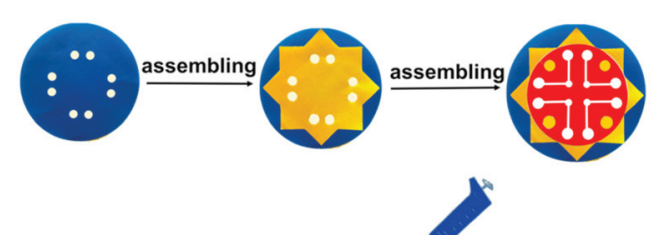

(B)

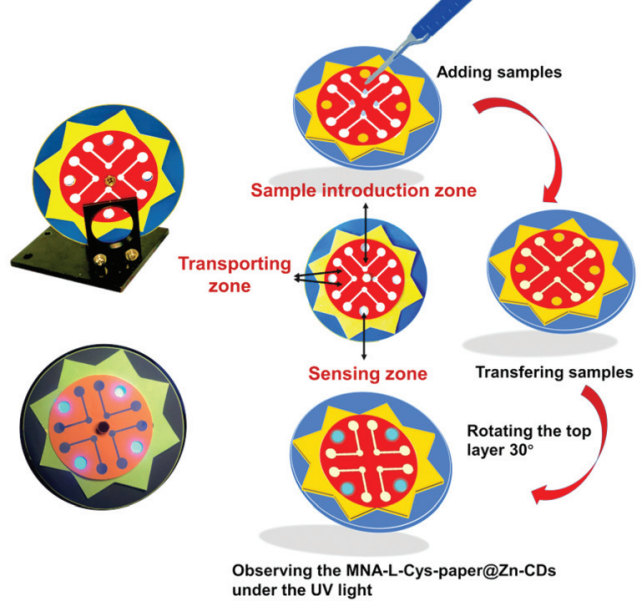

Fig. 1 The structure and detection process of the wheel type paperbased microfluidic chip. (A) Assembly process of the complete microfluidic chip. (B) The left side is a MNA-L-Cys-paper@Zn-CD $\mu$ PAD fixed on the sample holder and photograph of the chip under UV light (365 nm). The right side is the detection procedure of the $\mu \mathrm{PAD}$. different volumes $(4,5,6,7,8,910 \mathrm{~mL})$ of Zn-CDs $(12.5 \mathrm{mg}$ $\left.\mathrm{mL}^{-1}\right)$ to obtain paper@Zn-CDs. For volumes of MNA and L-Cys, MNA ( $2 \mathrm{mg} \mathrm{L}^{-1}$ ) and L-Cys $\left(1.6 \mathrm{mg} \mathrm{L}^{-1}\right)$ mixed solution $(0,8,16,24,32,40 \mathrm{~mL})$ was added and diluted with water to make up to $40 \mathrm{~mL}$ in the fabrication of MNA-L-Cys-paper@ZnCDs. As for $\mathrm{pH}$ values, $0.01 \mathrm{M}$ PBS buffer solution ( $\mathrm{pH}$ 5-9) was used to keep the $\mathrm{Cu}^{2+}$ concentration at $50 \mu \mathrm{g} \mathrm{L}^{-1}$. The fluorescence intensity under different conditions was measured following the method mentioned in Section 2.8.

\subsection{Detection of $\mathrm{Cu}^{2+}$ using MNA-L-Cys-paper@Zn-CD $\mu$ PAD and selectivity investigation}

The detection process of the $\mu \mathrm{PAD}$ is displayed in Fig. 1B. The top layer contained 4 sample introduction zones, which were connected to the transporting zones. The large circular sample zones of the top sampling layer overlapped with the holes of the middle isolation layer and the sensing sites of the bottom sensor layer (Fig. S1A $\dagger$ ). $20 \mu \mathrm{L}$ samples were added to the sampling zones and flowed along the hydrophilic " $L$ " channels to the sensing sites of the bottom sensor layer. After reaction between the samples and the MNA-L-Cys-paper@Zn-CDs on the sensing sites of the bottom sensor layer for $7 \mathrm{~min}$, we rotated the top layer by $30^{\circ}$ (Fig. $\mathrm{S} 1 \mathrm{~B} \dagger$ ). The holes of the top layer would be overlapped with the holes of the middle isolation layer and the sensing sites of the bottom sensor layer so that the sensing sites could be exposed. Then, the device was fixed on the sample holder of the fluorescence spectrophotometer to detect signals. Four blue fluorescence areas could be observed under a $365 \mathrm{~nm}$ ultraviolet lamp. Then, the $\mu$ PAD was fixed on the sample holder of the fluorescence spectrophotometer to detect signals at individual zones. The fluorescence intensity of the MNA-L-Cys-paper@Zn-CDs at $450 \mathrm{~nm}$ was recorded under an excitation wavelength of $354 \mathrm{~nm}$.

The selectivity of the MNA-L-Cys-paper@Zn-CD $\mu$ PAD was evaluated using metal ions that are commonly found in the water environment. $\mathrm{Cu}^{2+}$ ions were mixed with different metal ions $\left(\mathrm{Ca}^{2+}, \mathrm{Co}_{2}{ }^{+}, \mathrm{Cr}^{3+}, \mathrm{Fe}^{3+}, \mathrm{Mn}^{2+}, \mathrm{Na}^{+}, \mathrm{Ni}^{2+}, \mathrm{K}^{+}\right.$, and $\mathrm{Mg}^{2+}$ ), respectively. In the mixed solution, $\mathrm{Cu}^{2+}$ ions were at a concentration of $50 \mu \mathrm{g} \mathrm{L}^{-1}$, and interfering ions were at the same concentration of $250 \mu \mathrm{g} \mathrm{L} \mathrm{L}^{-1}$. In the absence of interfering ions, $\mathrm{Cu}^{2+}$ ions were also at a concentration of $50 \mu \mathrm{g} \mathrm{L}^{-1} .20 \mu \mathrm{L}$ of an interfering analyte was added to the sampling zone and determined following the method mentioned above. To investigate the stability of the MNA-L-Cys-paper@Zn-CD $\mu \mathrm{PAD}$, the fluorescence intensity of the chip was tested every 5 days. The MNA-L-Cys-paper@Zn-CD $\mu$ PAD was stored in the dark at $4{ }^{\circ} \mathrm{C}$.

\subsection{Analysis of real samples}

To evaluate the practicability of the MNA-L-Cys-paper@Zn-CD $\mu \mathrm{PAD}$, fluorescence analysis was carried out for the determination of $\mathrm{Cu}^{2+}$ in the Yangtze River, lake and tap water samples. $\mathrm{Cu}^{2+}$ in different concentrations $(0,2,10$, and $25 \mu \mathrm{g}$ $\mathrm{L}^{-1}$ ) was spiked on the MNA-L-Cys-paper@Zn-CD $\mu \mathrm{PAD}$, respectively. The contents of $\mathrm{Cu}^{2+}$ in the samples $(20 \mu \mathrm{L})$ were determined by fluorescence and inductively coupled plasma mass spectrometry (ICP-MS), respectively. 


\section{Results and discussion}

\subsection{Detection mechanism of MNA-L-Cys-paper@Zn-CD $\mu \mathrm{PAD}$}

The preparation process and sensing principle of the paperbased fluorescent sensor are shown in Fig. 2. First, in order to remove impurities and activate the hydroxyl group on the surface of bare paper, it was soaked in hydrochloric acid for 30 min. Then, APTES was added for the surface grafting of amino groups on the paper. The amide bonds were generated between the carboxyl groups of the Zn-CDs and the amino groups on the surface of the paper by EDC/NHS activation, thus enabling the stable grafting of the Zn-CDs on the paper. Then, MNA and L-Cys were added to fabricate the MNA-L-Cyspaper@Zn-CDs. When copper ions were added, the fluorescence of the MNA-L-Cys-paper@Zn-CDs was quenched by the formation of a nonfluorescent ground-state complex.

The possible mechanism of fluorescence quenching was investigated by fluorescence lifetime, UV absorption spectra and zeta potential in Fig. 3. The fluorescence lifetime of the MNA-L-Cys-Zn-CDs was $2.6 \mathrm{~ns}$ in the absence of $\mathrm{Cu}^{2+}$. However in the presence of $\mathrm{Cu}^{2+}\left(10,20,30,40\right.$, and $\left.50 \mu \mathrm{g} \mathrm{L}^{-1}\right)$, the fluorescence lifetimes were $2.4,2.7,2.8,2.6$, and $2.5 \mathrm{~ns}$, respectively (Fig. 3A). The fluorescence lifetime hardly changed, eliminating the possibility of dynamic quenching. ${ }^{35}$ To elucidate the mechanism of fluorescence quenching, the UV absorption spectra were obtained, as shown in Fig. 3B. The synthesized Zn-CDs exhibited an absorption peak at $227 \mathrm{~nm}$, which was ascribed to the $\pi-\pi^{*}$ transition of the $\mathrm{C}=\mathrm{C}$ group. ${ }^{36}$ The solution of MNA-L-Cys-Zn-CDs showed two absorption peaks at $300 \mathrm{~nm}$ and $347 \mathrm{~nm}$, respectively. In the presence of $\mathrm{Cu}^{2+}$, the absorption peak at $347 \mathrm{~nm}$ disappeared while the other peak diminished, indicating the formation of a ground-state complex between the MNA-L-Cys-Zn-CDs and $\mathrm{Cu}^{2+} \cdot{ }^{37}$ As shown in Fig. 3C, the zeta potential of MNA-L-Cys-Zn-CDs was $-1.26 \mathrm{mV}$, which was attributed to the carboxyl group on the surface of the MNA-L-Cys-Zn-CDs. When $\mathrm{Cu}^{2+}$ was added to the system, the zeta potential was $1.17 \mathrm{mV}$, which might be ascribed to the combination of the carboxyl group on the surface of the MNA-L-Cys-Zn-CDs with $\mathrm{Cu}^{2+}$. The above results
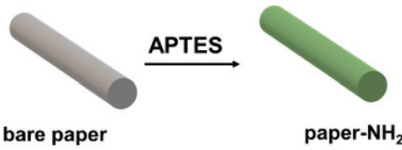

EDC/NHS

bare pape

paper- $\mathrm{NH}_{2}$

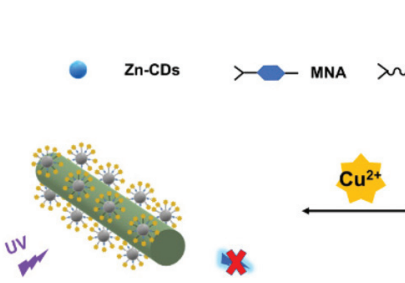

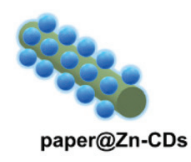
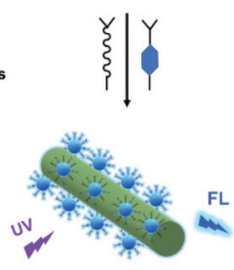

MNA-L-Cys-paper@Zn-CDs

Fig. 2 Schematic diagram of the preparation process and sensing principle of the MNA-L-Cys-paperaZn-CD $\mu$ PAD.
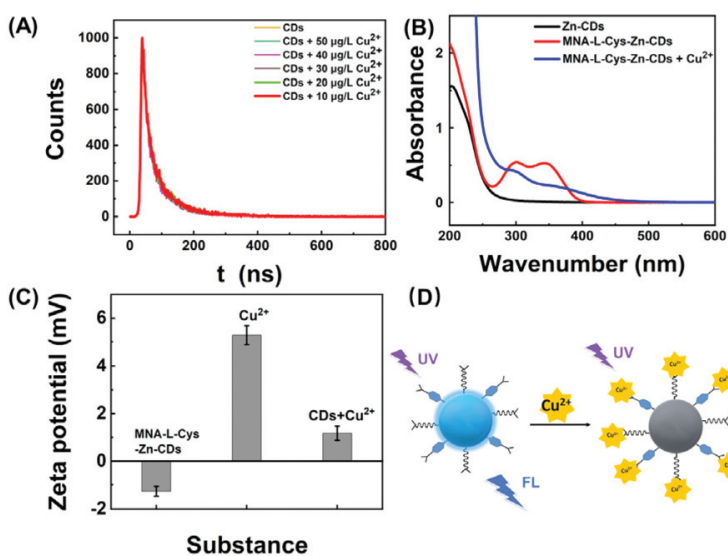

(D)

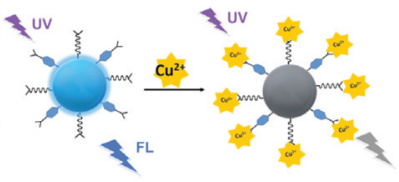

Fig. 3 (A) Fluorescence lifetimes of MNA-L-Cys-Zn-CDs with different concentrations of $\mathrm{Cu}^{2+}$. (B) UV absorption spectra of Zn-CDs and MNAL-Cys-Zn-CDs with different concentrations of $\mathrm{Cu}^{2+}$. (C) The zeta potential. (D) The schematic description of the static quenching.

proved that the mechanism of fluorescence quenching could be attributed to static quenching. ${ }^{35}$ Thus, the schematic mechanism can be described, as shown in Fig. 3D. MNA is an aromatic compound with an unsaturated six-membered heterocyclic ring containing sulfhydryl groups. The sulfhydryl group and nitrogen of MNA can interact with Zn-CDs, and the nitrogen and carboxyl groups on the other end of MNA can chelate with copper ions. If only MNA were added to paper@Zn-CDs, Zn-CDs would interact with S and N. This would greatly reduce the surface coverage efficiency of the $\mathrm{Zn}$ CDs, which was unfavorable for detecting $\mathrm{Cu}^{2+}$. While MNA and L-Cys containing sulfhydryl and carboxyl groups were simultaneously added to the paper@Zn-CDs, the linear L-Cys could competitively bind to Zn-CDs. Since MNA was in a vertical position on $\mathrm{Zn}$-CDs, it could release more $\mathrm{N}$ atoms and carboxyl groups to chelate with $\mathrm{Cu}^{2+}$ to form a nonfluorescent ground-state complex, causing fluorescence quenching. ${ }^{19}$ Thus, the sensitivity of detection was greatly improved.

\subsection{Characterization of Zn-CDs and the MNA-L-Cys- paper@Zn-CD $\mu$ PAD}

The synthesis of fluorescent materials is a fundamental part of the fluorescent sensor. In this work, Zn-CDs were prepared by pyrolysis. The HRTEM image of Zn-CDs is presented in Fig. 4A. Zn-CDs showed uniform spherical morphology in the diameter range of 2-7 $\mathrm{nm}$. The fluorescence spectra of aqueous solutions of $\mathrm{Zn}$-CDs were collected at room temperature, as shown in Fig. 4B. The Zn-CDs displayed the highest fluorescence (FL) intensity at $450 \mathrm{~nm}$ with the maximum excitation wavelength of $354 \mathrm{~nm}$. The effects of the concentration of Zn-CDs on FL intensity are shown in Fig. S2A.† The FL intensity monotonically increased with the decrease of the concentration of $\mathrm{Zn}$-CDs from 20 to $6.25 \mathrm{mg} \mathrm{mL} \mathrm{m}^{-1}$, and reached the peak at $6.25 \mathrm{mg} \mathrm{mL}^{-1}$. Afterwards, when the concentration continued to decrease, the FL intensity decreased. The emission of Zn-CDs was excitation-dependent. As the exci- 

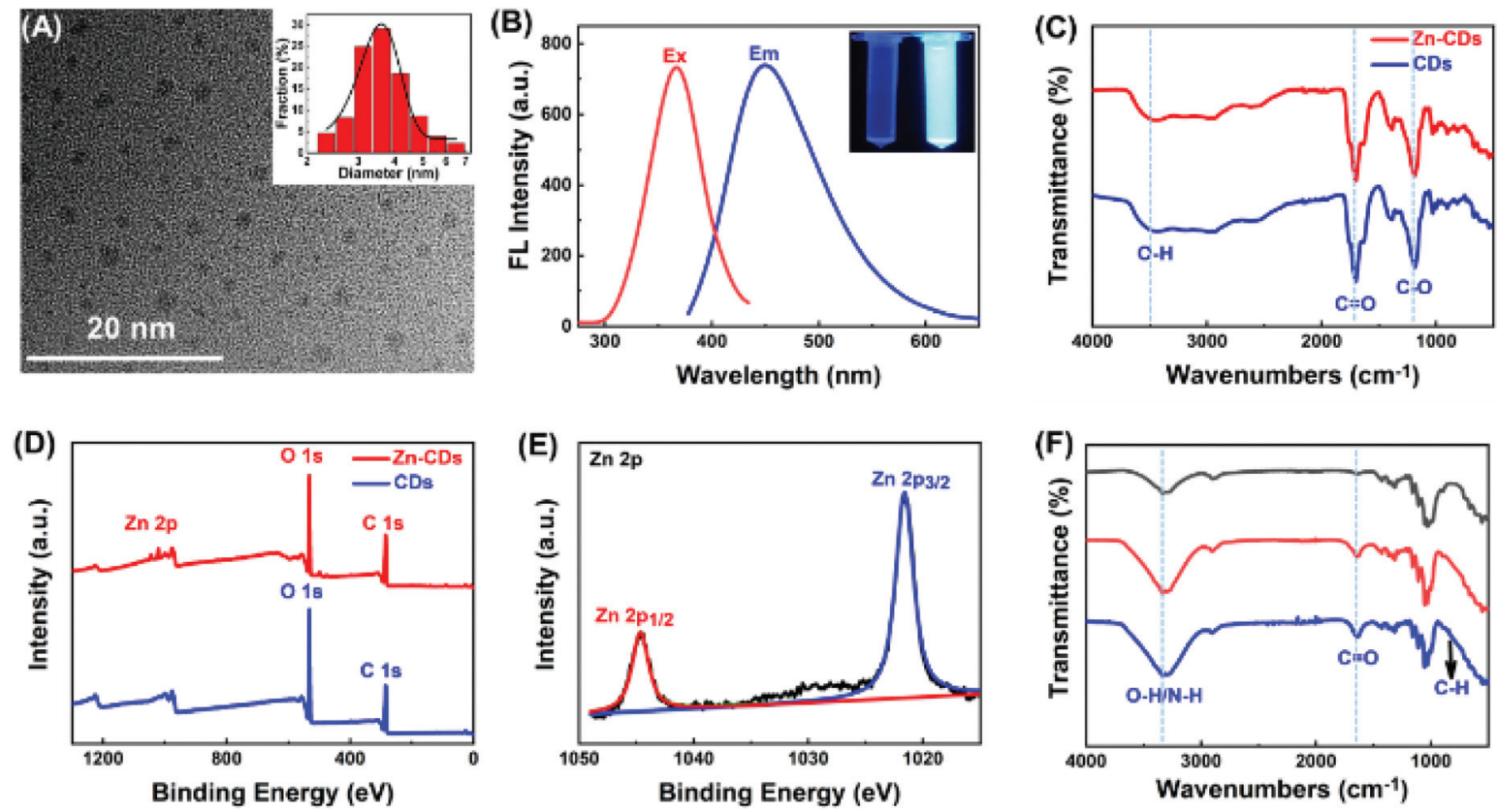

Fig. 4 (A) HRTEM and size distribution images. (B) Fluorescence spectra of $Z n-C D s$. (C) FT-IR spectra and (D) XPS spectra of Zn-CDs and CDs. (E) Zn $2 p$ spectrum of Zn-CDs. (F) FT-IR spectra of bare paper (black line), paper@Zn-CDs (red line) and MNA-L-Cys-paper(aZn-CDs (blue line). Inset of (B) shows the photo of water and $\mathrm{Zn}-\mathrm{CD}$ s under UV light $(365 \mathrm{~nm})$.

tation wavelength increased, the fluorescence peak shifted toward longer wavelengths, and the FL intensity declined (Fig. S2B $\dagger$ ).

The FT-IR spectra (Fig. 4C) of Zn-CDs (red curve) and CDs (blue curve) displayed a peak at around $3500 \mathrm{~cm}^{-1}$, which was attributed to the $\mathrm{O}-\mathrm{H}$ stretching vibration. ${ }^{38}$ The peak at $1713 \mathrm{~cm}^{-1}$ corresponded to the $\mathrm{C}=\mathrm{O}$ stretching vibration, and the peak at $1189 \mathrm{~cm}^{-1}$ belonged to the $\mathrm{C}-\mathrm{O}$ stretching vibration. ${ }^{4}$ These results indicated the presence of carboxyl and hydroxyl groups, leading to good water solubility of CDs and Zn-CDs. The elemental compositions of the as-prepared CDs and Zn-CDs were examined by XPS. The XPS survey spectra of the Zn-CDs (Fig. 4D, red curve) confirmed the presence of the elements $\mathrm{Zn}, \mathrm{O}$ and C, while that of CDs (Fig. 4D, blue curve) implied the presence of elements $\mathrm{O}$ and $\mathrm{C}$.

In the high-resolution $\mathrm{Zn} \mathrm{2p} \mathrm{spectrum,} \mathrm{the} \mathrm{peaks} \mathrm{at} 1021.6$ and $1044.7 \mathrm{eV}$ were ascribed to $\mathrm{Zn} 2 \mathrm{p}_{3 / 2}$ and $\mathrm{Zn} 2 \mathrm{p}_{1 / 2}$, respectively (Fig. 4E). ${ }^{39}$ The $\mathrm{C}$ 1s XPS spectrum (Fig. S3A $\dagger$ ) revealed two peaks located at 284.6 and $288.7 \mathrm{eV}$, which could be ascribed to $\mathrm{C}-\mathrm{C}$ and $\mathrm{C}=\mathrm{O}$, respectively. ${ }^{3}$ The XPS results confirmed the presence of carboxyl and hydroxyl groups in the $\mathrm{Zn}$ CDs. The structure and morphology of the fabricated MNA-LCys-paper@Zn-CDs were characterized by FTIR, SEM and fluorescence microscopy. The functional groups of paper, paper@Zn-CDs and MNA-L-Cys-paper@Zn-CDs were investigated by FTIR (Fig. 4F). The broad peak of $3000-3500 \mathrm{~cm}^{-1}$ was ascribed to the vibrational absorption band of $\mathrm{O}-\mathrm{H} / \mathrm{N}-\mathrm{H}$, and the absorption peak at around $1641 \mathrm{~cm}^{-1}$ represented $\mathrm{C}=\mathrm{O} .{ }^{40}$ Interestingly, the stretching vibrations of the $\mathrm{O}-\mathrm{H}$ and $\mathrm{C}=\mathrm{O}$ became stronger, indicating that $\mathrm{Zn}$-CDs have been intro- duced on the surface of the paper. The FTIR spectra revealed the MNA and L-Cys groups (Fig. S4†). The peaks at 2550-2700 $\mathrm{cm}^{-1}$ were ascribed to the vibrational absorption band of $\mathrm{S}-\mathrm{H}^{19}$ The peak for the aromatic hydrocarbon was located at $843 \mathrm{~cm}^{-1}$, which was also observed in MNA-L-Cyspaper@Zn-CDs, indicating that the paper@Zn-CDs had been successfully functionalized with L-Cys and MNA.

The SEM images of bare paper and MNA-L-Cys-paper@ZnCDs are shown in Fig. S5. $\dagger$ Bare paper presented a smooth and irregular dendritic fibrous structure (Fig. S5A $\dagger$ ). Compared to the bare paper, there was a dense coating on the surface of the paper fiber after grafting of CDs, suggesting that Zn-CDs had been successfully linked with the paper surface by amide bonds (Fig. S5B $\dagger$ ).

The fluorescence microscopy images are shown in Fig. S6. $\dagger$ Bare paper did not emit fluorescence. Paper@Zn-CDs showed strong fluorescence, but MNA-L-Cys-paper@Zn-CDs showed weaker fluorescence due to the covering of MNA and L-Cys on the surface of paper@Zn-CDs. To obtain a higher fluorescence signal for MNA-L-Cys-paper@Zn-CDs, the concentration and grafting time of $\mathrm{Zn}$-CDs were optimized to $12.5 \mathrm{mg} \mathrm{L}^{-1}$ and 5 h, respectively (Fig. S7†).

\subsection{Optimal conditions of the MNA-L-Cys-paper@Zn-CD $\mu \mathrm{PAD}$}

The effects of different experimental conditions on the fluorescence signal were investigated, including the precursor ratio of $\mathrm{Zn}$-CDs (the mass ratio of citric acid: zinc acetate), volume of $\mathrm{Zn}$-CDs, volume of APTES, volumes of MNA and L-Cys, $\mathrm{pH}$ value, and reaction time. The mass ratios of precursors (citric acid:zinc acetate) were investigated from $10: 0$ to $10: 5$ 

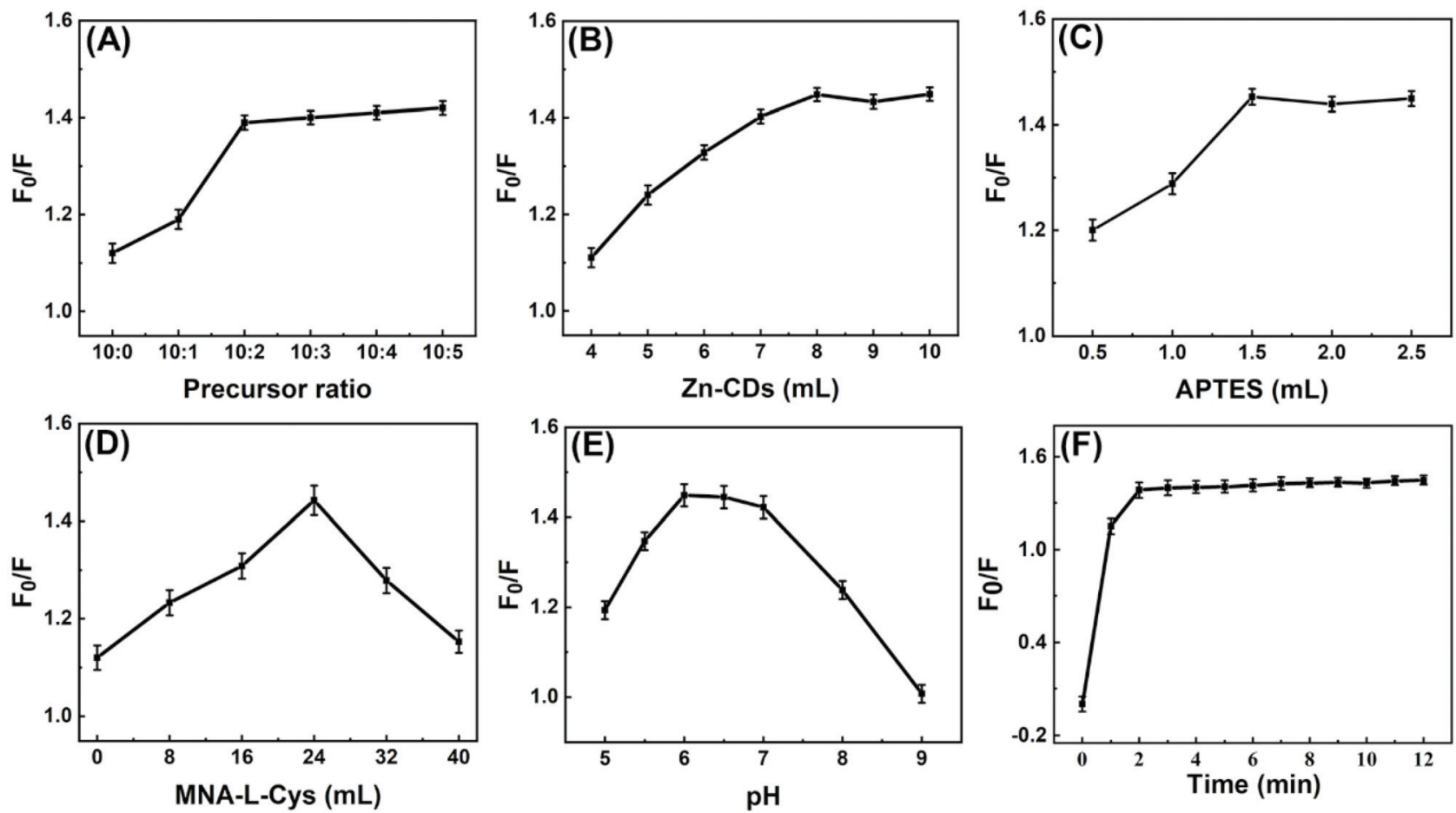

Fig. 5 Optimization of the experimental conditions: (A) precursor ratio of $\mathrm{Zn}-\mathrm{CDs}$ (the mass ratio of citric acid:zinc acetate), (B) volume of $\mathrm{Zn}$-CDs, (C) volume of APTES, (D) volume of MNA and L-Cys, (E) $\mathrm{pH}$ value, and (F) reaction time. The concentration of $\mathrm{Cu}^{2+}$ was $^{2} 50 \mathrm{~g} \mathrm{~L} \mathrm{~L}^{-1}$. The excitation wavelength and the emission wavelength were 354 and $450 \mathrm{~nm}$, respectively. The slit widths of emission and excitation were 5 and $1.5 \mathrm{~nm}(n=3)$.

(Fig. 5A). As proved by fluorescence spectra (Fig. S8†), Zndoping increased FL as well as the number of active sites for the attachment of MNA and L-Cys. Therefore, the fluorescence ratio increased when the precursor ratio ranged from 10:0 to $10: 2$ and achieved the maximum at $10: 2$. As the ratio continued to increase, the fluorescence ratio did not change much and the precursors could not react adequately. Therefore, $10: 2$ was selected as the best precursor ratio for $\mathrm{Cu}^{2+}$ detection.

The effects of the contents of Zn-CDs (4, 5, 6, 7, 8, 9, and $10 \mathrm{~mL}$ ) and APTES $(0.5,1.0,1.5,2.0$, and $2.5 \mathrm{~mL})$ were evaluated by detecting the fluorescence ratio. Fig. $5 \mathrm{~B}$ shows that the fluorescence ratio increased greatly with the increase of $\mathrm{Zn}$ CDs. The fluorescence ratio reached the maximum at $8 \mathrm{~mL} \mathrm{Zn-}$ CDs $\left(12.5 \mathrm{mg} \mathrm{mL}^{-1}\right)$. As the volume of $\mathrm{Zn}$-CDs continued to increase, the reaction reached saturation, and the fluorescence ratio remained nearly unchanged. Thus, $8 \mathrm{~mL}$ of Zn-CDs (12.5 $\mathrm{mg} \mathrm{mL} \mathrm{m}^{-1}$ ) was chosen for further experiments. Besides, the volume of APTES would affect the quality of amino groups grafted onto the paper. The fluorescence ratio rose from 0.5 to $1.5 \mathrm{~mL}$, and then remained almost constant from 1.5 to $2.5 \mathrm{~mL}$ (Fig. 5C). So, $1.5 \mathrm{~mL}$ was selected as the optimal amount of APTES.

The contents of MNA and L-Cys were also optimized. As illustrated in Fig. 5D, the fluorescence ratio increased when the volume of MNA and L-Cys increased from 0 to $24 \mathrm{~mL}$, and then decreased from 24 to $40 \mathrm{~mL}$. The quenching degree achieved was the maximum when the volume was $24 \mathrm{~mL}$.
Acidity had an important impact on fluorescence quenching. As shown in Fig. 5E, the quenching effect of the fluorescence signal increased rapidly when the solution $\mathrm{pH}$ increased from 5 to 6 , and reached the maximum at $\mathrm{pH}$ 6-7. Afterwards, the quenching effect of FL intensity exhibited a sharp decrease when the $\mathrm{pH}$ exceeded 7 . Under strongly acidic medium, the fluorescence intensity of Zn-CDs decreased, due to the formation of hydrogen bonds as well as protonation of the carboxyl group on the surface of the MNA-L-Cys-paper@ZnCDs. With the increase of the $\mathrm{pH}$ value, the groups of $\mathrm{L}$-Cys and MNA were deprotonated, which was able to strengthen the covalent binding force between the Zn-CDs and MNA-L-Cys. When the $\mathrm{pH}$ was higher than $7, \mathrm{Cu}(\mathrm{OH})_{2}$ precipitate would be produced, leading to a decrease of the fluorescence ratio. ${ }^{19,41}$ Given that the real samples were slightly acidic, pH 6.0 was selected as the optimum condition. The reaction time was also optimized, as shown in Fig. 5F. The fluorescence quenching signal increased rapidly for 0-2 min, and then changed slowly for 2-7 min. Afterwards, the quenching signal tended to be stable. Hence, $7 \mathrm{~min}$ was applied as the reaction time to ensure sufficient reactions.

\subsection{Detection performance of the MNA-L-Cys-paper@Zn-CD $\mu$ PAD}

The fluorescence performances of paper@Zn-CD $\mu \mathrm{PAD}$ and MNA-L-Cys-paper@Zn-CD $\mu$ PAD to $\mathrm{Cu}^{2+}$ are shown in Fig. 6 . Fig. 6A shows that the FL intensity of paper@Zn-CD $\mu$ PAD 

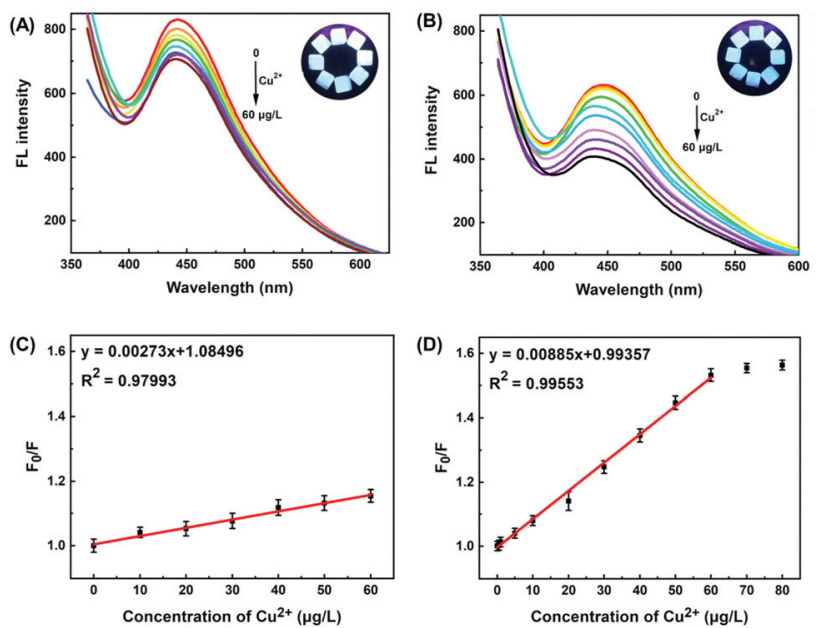

Fig. 6 Fluorescence spectra of (A) the paper@Zn-CD $\mu \mathrm{PAD}$, the concentrations of $\mathrm{Cu}^{2+}$ were $0,10,20,30,40,50$, and $60 \mu \mathrm{g} \mathrm{L}^{-1}$ and (B) the MNA-L-Cys-paperaZn-CD $\mu$ PAD, the concentrations of $\mathrm{Cu}^{2+}$ were 0 , $0.1,1,5,10,20,30,40,50$, and $60 \mu \mathrm{g} \mathrm{L}^{-1}$. The corresponding linear calibration plots of $(C)$ the paperaZn-CD $\mu \mathrm{PAD}$ and (D) the MNA-L-Cyspaper@Zn-CD $\mu \mathrm{PAD}$. The excitation wavelength and the emission wavelength were 354 and $450 \mathrm{~nm}$, respectively. The slit widths of emission and excitation were 5 and $1.5 \mathrm{~nm}(n=3)$. The inset photos show the corresponding color evolutions of the paper under a $365 \mathrm{~nm}$ UV lamp.

decreased with the increase of $\mathrm{Cu}^{2+}$ concentration $(0-60 \mu \mathrm{g}$ $\mathrm{L}^{-1}$ ). According to the Stern-Volmer equation: ${ }^{37}$

$$
F_{0} / F=1+K_{\mathrm{S}}[\mathrm{Q}]
$$

where $F_{0}$ and $F$ are the fluorescence intensities in the absence and presence of the quencher; $K_{S}$ is the ground state stability constant; and [Q] represents the concentration of the quencher. The fluorescence quenching degree $\left(F_{0} / F\right)$ was linearly related to the concentrations of $\mathrm{Cu}^{2+}$ (Fig. 6C). The calibration equation was $F_{0} / F=0.00273\left[\mathrm{Cu}^{2+}\right]+1.08296$, with a correlation coefficient $\left(R^{2}\right)$ of 0.979. Compared to the paper@Zn-CD $\mu \mathrm{PAD}$, the fluorescence quenching degree of the MNA-L-Cys-paper@Zn-CD $\mu$ PAD was much larger (Fig. 6B). The $F_{0} / F$ increased linearly with the concentrations of $\mathrm{Cu}^{2+}$ in the range from 0.1 to $60 \mu \mathrm{g} \mathrm{L} \mathrm{L}^{-1}$, which was described by the equation: $F_{0} / F=0.00885\left[\mathrm{Cu}^{2+}\right]+0.99357$, with a correlation coefficient $\left(R^{2}\right)$ of 0.996 (Fig. 6D). The limit of detection was $0.018 \mu \mathrm{g} \mathrm{L} \mathrm{L}^{-1}$ as calculated by 3 times the standard deviation rule $(3 \sigma / \mathrm{s})$, which was considerably lower than the maximum content $\left(1.3 \mathrm{mg} \mathrm{L} \mathrm{L}^{-1}\right)$ of $\mathrm{Cu}^{2+}$ stipulated by the U.S. Environmental Protection Agency. ${ }^{24}$ From Fig. $6 \mathrm{C}$ and D, we can see that the $K_{\mathrm{S}}$ of the MNA-L-Cys-paper@Zn-CD $\mu$ PAD was 0.00885 , which was higher than that of the paper@Zn-CD $\mu$ PAD. In addition, there were obvious differences in the changes in the fluorescence intensity between the paper@Zn$\mathrm{CD} \mu \mathrm{PAD}$ and the MNA-L-Cys-paper@Zn-CD $\mu \mathrm{PAD}$. In the presence of $\mathrm{Cu}^{2+}$ with the same concentration, the fluorescence quenching effect of the MNA-L-Cys-paper@Zn-CD $\mu$ PAD was larger than that of the paper@Zn-CD $\mu \mathrm{PAD}$ (Fig. 6A and B). So MNA-L-Cys-paper@Zn-CD $\mu$ PAD can detect a smaller change of the independent variable than the paper@Zn-CD $\mu \mathrm{PAD}$. All of these results indicated that the MNA-L-Cys-paper@Zn-CD $\mu \mathrm{PAD}$ displayed higher sensitivity than the paper@Zn-CD $\mu \mathrm{PAD}$.

\subsection{Selectivity and stability investigation of the MNA-L-Cys- paper@Zn-CD $\mu$ PAD}

To study the selectivity of the constructed sensor, we determined the signal response of the MNA-L-Cys-paper@Zn-CD $\mu \mathrm{PAD}$ to $\mathrm{Cu}^{2+}$ coexisting with impurities including $\mathrm{Ca}^{2+}, \mathrm{Co}_{2}{ }^{+}$, $\mathrm{Cr}^{3+}, \mathrm{Fe}^{3+}, \mathrm{Mn}^{2+}, \mathrm{Na}^{+}, \mathrm{Ni}^{2+}, \mathrm{K}^{+}$, and $\mathrm{Mg}^{2+}$. The concentrations of $\mathrm{Cu}^{2+}$ and other ions were $50 \mu \mathrm{g} \mathrm{L}^{-1}$ and $250 \mu \mathrm{g} \mathrm{L} \mathrm{L}^{-1}$, respectively. Other metal ions had no obvious interference effect on $\mathrm{Cu}^{2+}$ detection (Fig. 7A). The result revealed that the sensor showed great selectivity for $\mathrm{Cu}^{2+}$ detection.

The variability of sensing sites was also investigated. We randomly selected 10 different batches of MNA-L-Cyspaper@Zn-CDs, which were prefabricated under the same con-
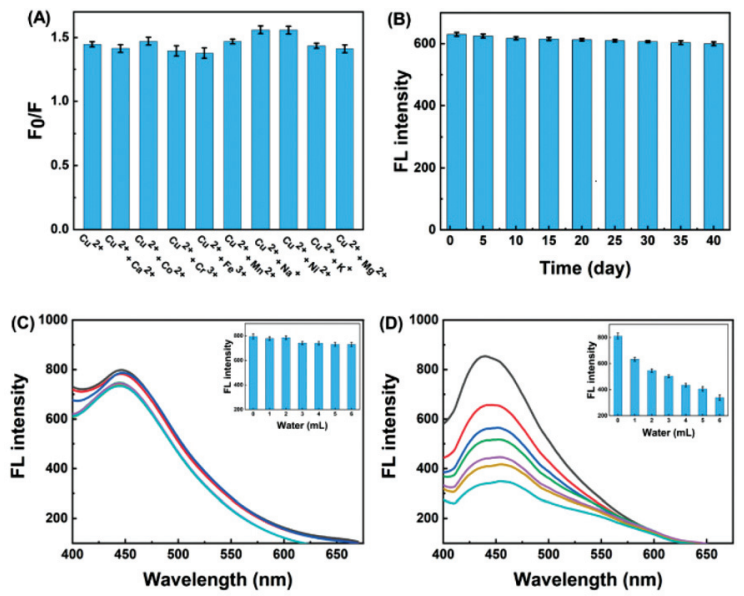

Fig. 7 (A) Selectivity of the MNA-L-Cys-paper@Zn-CD $\mu$ PAD. The concentrations of $\mathrm{Cu}^{2+}$ and interfering ions were $50 \mu \mathrm{g} \mathrm{L}^{-1}$ and $250 \mu \mathrm{g} \mathrm{L}^{-1}$ $(n=3)$. (B) Stability of the MNA-L-Cys-paperaZn-CD $\mu$ PAD $(n=3)$. Fluorescence spectra of (C) paper(aZn-CDs and (D) test strip washed with different volumes of water. The inset photos show the corresponding column charts.

Table 1 Determination of $\mathrm{Cu}^{2+}$ in tap water, lake water and Yangtze River water based on MNA-L-Cys-paper@Zn-CD $\mu$ PADs

\begin{tabular}{llcllr}
\hline Sample $(20 \mu \mathrm{L})$ & $\begin{array}{l}\text { Added } \\
\left(\mu \mathrm{g} \mathrm{L}^{-1}\right)\end{array}$ & $\begin{array}{l}\text { Founded } \\
\left(\mu \mathrm{g} \mathrm{L}^{-1}\right)\end{array}$ & $\begin{array}{l}\text { Recovery } \\
(\%)(n=5)\end{array}$ & $\begin{array}{l}\text { RSD }(\%) \\
(n=5)\end{array}$ & $\begin{array}{l}\text { ICP-MS } \\
\left(\mu \mathrm{g} \mathrm{L}^{-1}\right)\end{array}$ \\
\hline Tap water & 0 & 0.21 & - & 4.4 & 0.32 \\
& 2 & 2.11 & 105.5 & 6.3 & 2.48 \\
& 10 & 10.15 & 101.5 & 3.4 & 10.27 \\
Lake water & 25 & 26.7 & 106.8 & 3.7 & 25.63 \\
& 0 & 0.53 & - & 5.2 & 0.46 \\
& 2 & 2.17 & 108.5 & 6.5 & 2.32 \\
Yangtze river & 10 & 10.47 & 104.7 & 5.6 & 10.54 \\
water & 25 & 26.10 & 104.4 & 4.5 & 25.58 \\
& 2 & 0.87 & - & 3.8 & 0.79 \\
& 10 & 2.18 & 109.2 & 5.2 & 2.24 \\
& 25 & 26.38 & 103.8 & 4.8 & 10.57 \\
& & & 106. & 6.3 & 26.88
\end{tabular}


Table 2 Comparison of different methods for $\mathrm{Cu}^{2+}$ ion sensing

\begin{tabular}{|c|c|c|c|c|}
\hline Material & Detected method & Linear range $\left(\mu \mathrm{g} \mathrm{L}{ }^{-1}\right)$ & Detection limit $\left(\mu g \mathrm{~L}^{-1}\right)$ & Ref. \\
\hline Paper@QDs@Cu-IIPs & Fluorimetry & $0.11-58$ & 0.035 & 17 \\
\hline MNA-L-Cys-Zns : Mn(II)QDs & Fluorimetry & $0.32-32$ & 0.077 & 19 \\
\hline Carbon dots $/ \mathrm{Zn}(\mathrm{OH})_{2}$ & Fluorimetry & $0-38$ & 0.019 & 39 \\
\hline Dye-coded AgNPs & SERS $^{a}$ & - & 0.00064 & 42 \\
\hline Ibuprofen copped HgNPs & Voltammetry & - & 0.035 & 43 \\
\hline Au-AgNCs & Fluorimetry & $1.2-38.4$ & 0.448 & 44 \\
\hline GSH-AuNCs & Fluorimetry & $3.2-960$ & 1.86 & 45 \\
\hline MNA-L-Cys-paper@Zn-CDs & Fluorimetry & $0.1-60$ & 0.018 & This work \\
\hline
\end{tabular}

ditions, as sensing sites for fluorescence detection (Fig. S1D $\dagger$ ). As shown in Fig. S9, $\dagger$ the fluorescence intensity of the 10 different sensing sites did not show much difference (RSD of $2.8 \%$ ).

In order to investigate the stability of the MNA-L-Cyspaper@Zn-CD $\mu \mathrm{PAD}$, the FL intensity of the $\mu \mathrm{PAD}$ maintained for different days $(0,5,10,15,20,25,30,35$, and 40 days) was analyzed. The FL intensity showed little change (Fig. 7B). Thus, the MNA-L-Cys-paper@Zn-CD $\mu \mathrm{PAD}$ has good storage stability in the dark at $4{ }^{\circ} \mathrm{C}$. Furthermore, bare paper was impregnated with the solution of Zn-CDs (12.5 $\mathrm{mg} \mathrm{mL} \mathrm{mL}^{-1}$ ) directly to prepare a test strip. The test strip and paper@ZnCDs were washed with different volumes of water. The changes in FL intensity of the test strip and paper@Zn-CDs are presented in Fig. 7C and D. The FL intensity of the test strip dramatically decreased with increasing volumes of water, while that of paper@Zn-CDs only displayed a slight change. The results indicated that $\mathrm{Zn}$-CDs anchored on the paper through the amide bonds had better stability compared with the conventional immersion method.

\subsection{Practical application to real samples}

To verify the practical performance of the MNA-L-Cyspaper@Zn-CD $\mu \mathrm{PAD}$, we used the fabricated fluorescent sensor to detect $\mathrm{Cu}^{2+}$ in real water samples. Different concentrations of $\mathrm{Cu}^{2+}$ were added to real samples. The result is displayed in Table 1. The recoveries for $\mathrm{Cu}^{2+}$ in the water samples were between $98.1 \%$ and $115 \%$, and the relative standard deviations (RSDs) ranged from 3.4 to $6.5 \%$. The contents of $\mathrm{Cu}^{2+}$ were also detected by ICP-MS, which showed similar results to this method. Therefore, the MNA-L-Cys-paper@Zn-CD $\mu$ PAD can be used to measure the amount of $\mathrm{Cu}^{2+}$ in a complex water environment with high sensitivity.

\subsection{Comparison of different approaches}

The performance of the prefabricated MNA-L-Cys-paper@Zn$\mathrm{CD} \mu \mathrm{PAD}$ and previously reported methods for $\mathrm{Cu}^{2+}$ determination is presented in Table 2. The proposed method shows lower detection limit and wider detection range compared with the reported analysis methods which were based on SERS, ${ }^{42}$ voltammetry, ${ }^{43}$ and fluorimetry. ${ }^{17,19,39,44,45}$

\section{Conclusion}

In conclusion, a three-dimensional wheel type $\mu \mathrm{PAD}$ based on Zn-CDs decorated with MNA and L-Cys has been successfully fabricated for highly sensitive fluorescence detection of $\mathrm{Cu}^{2+}$. Zn-CDs were first firmly grafted on the paper through the amide bonds between the carboxyl groups of Zn-CDs and the amino groups of the paper. According to a series of experimental investigations, it has been verified that the Zn-CDs can be easily modified with MNA and L-Cys through coordination bonds, which is of significance in secondary functionalization of CDs. Compared to CDs, Zn-CDs can offer more active sites for the modification of MNA and L-Cys. The three-dimensional wheel type $\mu \mathrm{PAD}$ can detect multiple samples with different concentrations and is expected to detect different kinds of samples simultaneously. Moreover, the $\mu \mathrm{PAD}$ only requires a one-step rotation operation without taking out and cleaning, thus enhancing the detection efficiency. The constructed paper-based sensor presents high sensitivity, good selectivity and rapid response time for $\mathrm{Cu}^{2+}$ determination which might have potential prospects for environmental analysis and pointof-care testing.

\section{Conflicts of interest}

The authors declare no competing financial interest.

\section{Acknowledgements}

This work was supported by the National Natural Science Foundation of China (No. 21974085, 61971274, 62171268 and 22106179) and the China Postdoctoral Science Foundation (2021M693208).

\section{References}

1 Y. J. Wang, J. Chen, L. M. Liu, X. X. Xi, Y. M. Li, Z. L. Geng, G. Y. Jiang and Z. Zhao, Nanoscale, 2019, 11, 1618-1625.

2 Y. X. Xu, P. P. Li, D. Cheng, C. Y. Wu, Q. J. Lu, W. P. Yang, X. H. Zhu, P. Yin, M. L. Liu, H. T. Li and Y. Y. Zhang, J. Mater. Chem. B, 2020, 8, 10290-10308. 
3 P. P. Li, C. Y. Wu, Y. X. Xu, D. Cheng, Q. J. Lu, J. Gao, W. P. Yang, X. H. Zhu, M. L. Liu, H. T. Li, P. Yin and Y. Y. Zhang, Trends Anal. Chem., 2020, 131, 116007.

4 J. H. Guo, W. J. Lu, H. L. Zhang, Y. T. Meng, F. F. Du, S. M. Shuang and C. Dong, Sens. Actuators, B, 2021, 330, 129360.

5 F. T. Wang, L. N. Wang, J. Xu, K. J. Huang and X. Wang, Analyst, 2021, 146, 4418-4435.

6 R. S. Li, P. F. Gao, H. Z. Zhang, L. L. Zheng, C. M. Li, J. Wang, Y. F. Li, F. Liu, N. Li and C. Z. Huang, Chem. Sci., 2017, 8, 6829-6835.

7 B. B. Chen, M. L. Liu, C. M. Li and C. Z. Huang, Adv. Colloid Interface Sci., 2019, 270, 165-190.

8 T. Liu, N. Li, J. X. Dong, H. Q. Luo and N. B. Li, Sens. Actuators, B, 2016, 231, 147-153.

9 Q. Xu, P. Pu, J. G. Zhao, C. B. Dong, C. Gao, Y. S. Chen, J. R. Chen, Y. Liu and H. J. Zhou, J. Mater. Chem. A, 2015, 3, 542-546.

10 Y. F. Feng, Y. G. Li, S. Y. Yu, Q. R. Yang, Y. B. Tong and B. C. Ye, Analyst, 2021, 146, 5135-5142.

11 Z. T. Fan, Y. C. Li, X. H. Li, L. Z. Fan, S. X. Zhou, D. C. Fang and S. H. Yang, Carbon, 2014, 70, 149-156.

12 J. H. Deng, Q. J. Lu, Y. X. Hou, M. L. Liu, H. T. Li, Y. Y. Zhang and S. Z. Yao, Anal. Chem., 2015, 87, 2195-2203.

13 S. K. Tammina, Y. Wan, Y. Y. Li and Y. L. Yang, J. Photochem. Photobiol., B, 2020, 202, 111734.

14 Z. Y. Ma, Y. Sun, J. W. Xie, P. P. Li, Q. J. Lu, M. L. Liu, P. Yin, H. Li, Y. Y. Zhang and S. Z. Yao, ACS Appl. Mater. Interfaces, 2020, 12, 15919-15927.

15 Z. Y. Ma, Y. F. Xu, P. P. Li, D. Cheng, X. H. Zhu, M. L. Liu, Y. Y. Zhang, Y. Liu and S. Z. Yao, Anal. Chem., 2021, 93, 3586-3593.

16 K. Y. Yi, X. T. Zhang and L. Zhang, Sci. Total Environ., 2020, 743, 140692.

17 J. Qi, B. W. Li, X. R. Wang, Z. Zhang, Z. Wang, J. L. Han and L. X. Chen, Sens. Actuators, B, 2017, 251, 224-233.

18 Q. Xu, Y. Liu, R. G. Su, L. L. Cai, B. F. Li, Y. Y. Zhang, L. Z. Zhang, Y. J. Wang, Y. Wang, N. Li, X. Gong, Z. P. Gu, Y. S. Chen, Y. L. Tan, C. B. Dong and T. S. Sreeprasad, Nanoscale, 2016, 8, 17919-17927.

19 J. R. Wang, J. L. Yu, X. Y. Wang, L. Y. Wang, B. W. Li, D. Z. Shen, Q. Kang and L. Chen, Microchim. Acta, 2018, 185, 420.

20 L. K. Kumawat, N. Mergu, A. K. Singh and V. K. Gupta, Sens. Actuators, B, 2015, 212, 389-394.

21 G. Sivaraman, M. Iniya, T. Anand, N. G. Kotla, O. Sunnapu, S. Singaravadivel, A. Gulyani and D. Chellappa, Coord. Chem. Rev., 2018, 357, 50-104.

22 D. Huster and S. Lutsenko, Mol. Biosyst., 2007, 3, 816-824.

23 L. J. Hayward, J. A. Rodriguez, J. W. Kim, A. Tiwari, J. J. Goto, D. E. Cabelli, J. S. Valentine and R. H. Brown Jr., J. Biol. Chem., 2002, 277, 15923-15931.
24 J. W. Liu and Y. Lu, J. Am. Chem. Soc., 2007, 129, 98389839.

25 X. L. Shao, H. Gu, Z. Wang, X. L. Chai, Y. Tian and G. Y. Shi, Anal. Chem., 2013, 85, 418-425.

26 W. R. Yang, D. Jaramillo, J. J. Gooding, D. B. Hibbert, R. Zhang, G. D. Willett and K. J. Fisher, Chem. Commun., 2001, 1982-1983.

27 M. Zhou, L. Han, D. M. Deng, Z. Zhang, H. B. He, L. Zhang and L. Q. Luo, Sens. Actuators, B, 2019, 291, 164-169.

28 N. Mergu and V. K. Gupta, Sens. Actuators, B, 2015, 210, 408-417.

29 M. R. Awual and M. M. Hasan, Sens. Actuators, B, 2015, 206, 692-700.

30 X. X. He, J. Zhang, X. G. Liu, L. Dong, D. Li, H. Y. Qiu and S. C. Yin, Sens. Actuators, B, 2014, 192, 29-35.

31 H. Ding, C. S. Liang, K. B. Sun, H. Wang, J. K. Hiltunen, Z. J. Chen and J. C. Shen, Biosens. Bioelectron., 2014, 59, 216-220.

32 J. Qi, B. W. Li, X. Y. Wang, L. W. Fu, L. Q. Luo and L. X. Chen, Anal. Chem., 2018, 90, 11827-11834.

33 H. X. Qin, J. T. Ren, J. H. Wang and E. K. Wang, Chem. Commun., 2010, 46, 7385-7387.

34 Y. Y. Han, Y. Li, W. Si, D. Wei, Z. X. Yao, X. P. Zheng, B. Du and Q. Wei, Spectrochim. Acta, Part A, 2011, 79, 1546-1551.

35 F. L. Zu, F. Y. Yan, Z. J. Bai, J. X. Xu, Y. Y. Wang, Y. C. Huang and X. G. Zhou, Microchim. Acta, 2017, 184, 1899-1914.

36 Z. Y. Gan, X. T. Hu, X. W. Huang, Z. H. Li, X. B. Zou, J. Y. Shi, W. Zhang, Y. X. Li and Y. W. Xu, Sens. Actuators, B, 2021, 328, 128997.

37 K. Patir and S. K. Gogoi, Nanoscale Adv., 2019, 1, 592-601.

38 H. J. Qi, M. Teng, M. Liu, S. X. Liu, J. Li, H. P. Yu, C. B. Teng, Z. H. Huang, H. Liu, Q. Shao, A. Umar, T. Ding, Q. Gao and Z. H. Guo, J. Colloid Interface Sci., 2019, 539, 332-341.

39 J. Hou, T. Y. Zhou, L. Wang, P. Zhang and L. Ding, Sens. Actuators, B, 2016, 230, 615-622.

40 Z. Han, D. Y. Nan, H. Yang, Q. Q. Sun, S. Pan, H. Liu and X. L. Hu, Sens. Actuators, B, 2019, 298, 126842.

41 H. Z. Lu, S. F. Xu and J. Q. Liu, ACS Sens., 2019, 4, 19171924.

42 F. Li, J. Wang, Y. M. Lai, C. Wu, S. Q. Sun, Y. H. He and H. Ma, Biosens. Bioelectron., 2013, 39, 82-87.

43 Z. A. Tagar, Sirajuddin, N. Memon, M. S. Kalhoro, P. O’Brien, M. A. Malik, M. I. Abro, S. S. Hassan, N. H. Kalwar and Y. Junejo, Sens. Actuators, B, 2012, 173, 745-751.

44 E. Babaee, A. Barati, M. B. Gholivand, A. A. Taherpour, N. Zolfaghar and M. Shamsipur, J. Hazard. Mater., 2019, 367, 437-446.

45 X. G. Chen, Q. J. Lu, D. Liu, C. Y. Wu, M. L. Liu, H. T. Li, Y. Y. Zhang and S. Z. Yao, Microchim. Acta, 2018, 185, 188. 